\title{
Implantation, Sputtering and Electromigration: Kelvin Probe Microscopy of Focussed Ion Beam Processed SIMOX.
}

\author{
Marion A. Stevens-Kalceff ${ }^{1,2}$ and T.L. Sobey ${ }^{1}$ \\ ${ }^{1}$ School of Physics, University of New South Wales, Sydney, 2052 NSW Australia. \\ ${ }^{2}$ Electron Microscope Unit, University of New South Wales, Sydney, 2052 NSW Australia.
}

Scanning Probe Microscopy techniques provide three dimensional nanometer scale spatial resolution information about the structural, mechanical and chemical properties of surfaces and nanoscale materials. Kelvin Probe Microscopy (KPM) is a specialized Atomic Force Microscopy technique in which long-range Coulomb forces between a conductive atomic force probe and a specimen enable the electrical potential at the specimen surface to be characterized.[1] The surface potential may be associated with a charge distribution within the specimen, and information about the spatial extent and relative magnitude of this charge distribution can be deduced from the surface potential data.[2]

The Separation by IMplantation of OXygen or SIMOX approach is a popular method for fabricating Silicon-On-Insulator (SOI) wafers for application in high-performance/ low power CMOS and MEMS devices. Oxygen ions are implanted into a silicon substrate which is then annealed at high temperature in an Ar- $\mathrm{O}_{2}$ atmosphere to form a Buried OXide (BOX) layer. The SIMOX process produces very uniform SOI and BOX layers with reduced defect densities. The SIMOX wafer used in this investigation has a SOI top layer thickness of $\sim 220 \mathrm{~nm}$ and a BOX layer thickness of $\sim 400 \pm$ $10 \mathrm{~nm}$ on a p-type silicon (100) substrate.[3]

The SIMOX specimen was irradiated with a continuous normal incident $30 \mathrm{keV}$ focussed $\mathrm{Ga}^{+}$ion beam for increasing exposure times. Focussed ion irradiation of materials used in semiconductor technology is of great importance in fundamental physics (scattering, impurity effects) and practical applications (nano-modification, depth resolved analysis, TEM sample preparation).[4] A Veeco/ Digital Instruments extended Dimension 3000 Scanning Probe Microscope operating in ambient Kelvin probe mode, has been used to characterize residual potentials associated with the SIMOX specimen as it is processed with a $30 \mathrm{keV}, 6600 \mathrm{pA}$ focussed $\mathrm{Ga}^{+}$ion beam in an FEI xP200 Focused Ion Beam system. Irradiation induced trapped charge produces electric fields within the implanted SIMOX. The charging processes are localized, dynamic, and are dependent on pre-existing and irradiation induced defect concentrations. The reproducible characteristic surface potentials associated with the $\mathrm{Ga}^{+}$irradiated SIMOX have been measured using Kelvin Probe Microscopy. Surface potentials of a few hundred $\mathrm{mV}$ are measured and are compared with Electron Microprobe data of the $\mathrm{Ga}, \mathrm{O}$ and $\mathrm{Si}$ concentrations. The high spatial correlation between the measured potential and the oxygen concentration indicates irradiation induced electromigation of oxygen to the periphery of the milled regions of the specimens. The measured characteristic surface potentials are compared with model surface potentials calculated using three-dimensional conformal Finite Element Analysis.[5] The observed potentials are associated with a number of complex competing physical processes including ion neutralisation, positive/secondary ion emission/ sputtering, ion implantation and charge trapping resulting in local electromigration of oxygen. This investigation shows that ion beam processing of SIMOX can result in local modification of the chemical composition of the specimen via implantation and electromigration. This has implications for the processing of semiconductor materials in a Focussed Ion Beam system. 


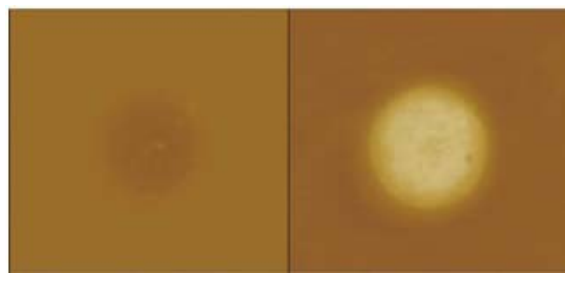

(a) SIMOX sputtered to a depth of $\sim 80 \mathrm{~nm}$ into the Silicon on Insulator (SOI) layer.

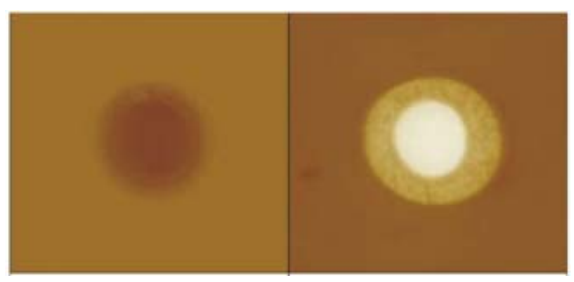

(b) SIMOX sputtered to a depth of $\sim 260 \mathrm{~nm}$ through the Silicon on Insulator (SOI) layer and into the Buried Oxide (BOX) layer.

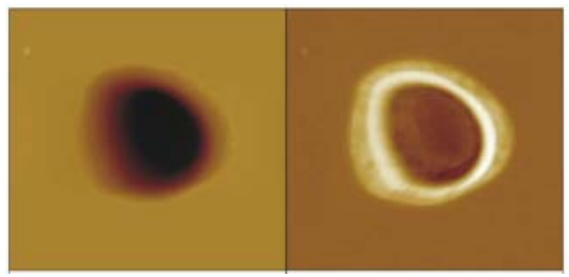

(c) SIMOX sputtered to a depth of $\sim 1270 \mathrm{~nm}$ through the Silicon on Insulator (SOI) layer and the Buried Oxide (BOX) layer and into the pSilicon (100) substrate.

FIG. 1. Typical $20 \times 20 \mu \mathrm{m}^{2}$ topography and corresponding surface potential images from the SIMOX specimen irradiated with a stationary normal incidence $30 \mathrm{keV}$ focussed $\mathrm{Ga}^{+}$ion beam for increasing exposure times and therefore increasing sputtered depths of (a) 80nm, (b) 260nm and (c) $\sim 1270 \mathrm{~nm}$. The SIMOX wafer has a SOI top layer thickness of $\sim 220 \mathrm{~nm}$ and a BOX layer thickness of $\sim 400 \pm 10 \mathrm{~nm}$ on a p-type silicon (100) substrate.

\section{References}

[1] Bonnell, D. A. Scanning Probe Microscopy and Spectroscopy: Theory, Techniques and Applications. Wiley-VCH. New York, 2001.

[2] Stevens-Kalceff, M. A. Microscopy and Microanalysis 10, (2004) 797-803.

[3] SIMOX 400 wafer (www.ibis.com)

[3] Stevens-Kalceff, M. A. Physical Review B. 57, (1998) 5674-5683.

[4] International Technology Roadmap for Semiconductors (2004) http://public.itrs.net

[5] Programs HiPhi and Metamesh, Field Precision, Albuquerque, NM

[6] Support from the Australian Research Council and the Electron Microscope Unit at University of New South Wales is gratefully acknowledged.

Contact author: Marion.Stevens-Kalceff@unsw.edu.au 